\title{
CARACTERÍSTICAS FÍSICAS E MECÂNICAS DO ENDOCARPO E DA AMÊNDOA DA CASTANHA DE CAJU 'CCP 76' ANTES E APÓS TRATAMENTO TÉRMICO'
}

\author{
MAX C. DE ARAÚJO ${ }^{2}$, ANTONIO C. DE O. FERRAZ ${ }^{3}$
}

\begin{abstract}
RESUMO: As características viscoelásticas da casca da castanha de caju in natura dificultam a sua decorticação por compressão. Para facilitar a abertura da casca e a liberação da amêndoa, as castanhas são submetidas à hidratação e tratamento térmico em banho de líquido da casca da castanha (LCC) a $210^{\circ} \mathrm{C}$. Com o objetivo de conhecer o comportamento do produto, para futuros desenvolvimentos de mecanismos decorticadores, procurou-se caracterizar a castanha do clone 'CCP 76' por meio das principais dimensões, massa, volume e ensaios mecânicos do endocarpo e da amêndoa, antes e após o tratamento térmico. Os tratamentos consistiram em várias combinações de tempo de hidratação e tempo de residência em banho de LCC. Aplicou-se a metodologia de superfície de resposta para identificar o melhor tratamento. Construíram-se dispositivos de abertura manual da castanha, cisalhamento do endocarpo e penetração da amêndoa para a realização dos ensaios mecânicos. Observaram-se alterações no comprimento, na largura e na espessura, assim como na massa e no volume, antes e após os tratamentos. Os resultados do ensaio de cisalhamento do endocarpo e da rigidez da amêndoa indicam diferença entre o material in natura e o tratado termicamente. As alterações nas dimensões de massa e de volume apontam para a necessidade de uma classificação após o tratamento térmico, quando essas constituírem parâmetros relevantes no processo de decorticação. A tendência da superfície de resposta mostrou que o ensaio com $79 \mathrm{~h}$ de hidratação e $165 \mathrm{~s}$ de residência em LCC a $210^{\circ} \mathrm{C}$ foi o mais próximo da região de ótimo.
\end{abstract}

PALAVRAS-CHAVE: Anacardium ocidentale, viscoelasticidade, propriedades mecânicas.

\section{MECHANICAL CHARACTERISTICS OF ENDOCARP AND KERNEL OF CASHEW NUT 'CCP 76' PRIOR AND POST THERMAL TREATMENT}

\begin{abstract}
Viscoelastic characteristics of raw cashew nut shell hinder its decortication by compression. In order to facilitate the shell opening and to liberate the kernel, nuts were subjected to hydration and thermal treatment in cashew nut shell liquid (CSL) at $210^{\circ} \mathrm{C}$. With the objective of developing more appropriate shelling mechanisms the cashew nut 'CCP 76' was characterized by its main dimensions, mass and volume, as well as mechanical behavior of the endocarp and kernel, prior and after thermal treatments. The treatments consisted in submitting the nuts to several combinations of hydration and thermal treatment times. The response surface methodology was applied to identify the best treatment. Specially built devices were used for manual opening of the cashew nut, shear of the endocarp and penetration of the kernel. Changes were observed in the dimensions, length, width, and thickness as well as in mass and volume values prior and after treatments. Results of endocarp shear and kernel stiffness tests pointed out differences between raw and treated nuts. Changes in dimensions, mass, and volume pointed out to performing sizing after thermal treatments if dimensions are relevant in shelling. The response surface methodology indicated that the treatment comprising $79 \mathrm{~h}$ hydration and $165 \mathrm{~s}$ of residence in CSL at $210{ }^{\circ} \mathrm{C}$ was the closest to the optimum region.
\end{abstract}

KEYWORDS: Anacardium ocidentale, viscoelasticity, mechanical properties.

\footnotetext{
${ }^{1}$ Extraído da Tese de Doutorado do primeiro autor.

${ }^{2}$ Professor Adjunto I, Departamento de Engenharia Agrícola e Solos, CCA, Universidade Federal do Piauí, max@ufpi.br

${ }^{3}$ Professor Livre-Docente, Faculdade de Engenharia Agrícola, UNICAMP, carlos@agr.unicamp.br

Recebido pelo Conselho Editorial em: 19-6-2006

Aprovado pelo Conselho Editorial em: 12-6-2008
} 


\section{INTRODUÇÃO}

A castanha de caju in natura apresenta características viscoelásticas que dificultam a sua decorticação por compressão, pois tende a absorver a energia por meio da deformação do pericarpo e da amêndoa. Para modificar essas características, prepara-se a castanha por meio de hidratação e de tratamento térmico, tornando a casca mais frágil e, portanto, mais fácil de romper com aplicação de pequena deformação.

O tratamento térmico, no entanto, não deve afetar a amêndoa, evitando o seu escurecimento e conseqüente depreciação. O tratamento térmico, por meio do banho das castanhas no próprio líquido da casca (LCC) em alta temperatura, realizado na indústria de beneficiamento, tem como objetivo, além de tornar a casca frágil, promover uma folga (espaço) entre a casca e a amêndoa. Os parâmetros de controle mais importantes envolvidos no tratamento térmico são o teor de água da castanha, a temperatura e o tempo de residência. As transferências de calor e massa no processo são muito complexas e, devido à geometria e à distribuição irregular de massa da castanha, fica difícil obter um tratamento térmico uniforme. Assim, as extremidades aquecem-se mais rapidamente que o restante da amêndoa.

Uma situação ideal seria a de um corpo perfeitamente esférico, onde as trocas de calor ocorressem de maneira uniforme. BALASUBRAMANIAN (2001) relata resultados da determinação de algumas propriedades físicas da castanha in natura, tais como: massa, porosidade, densidade aparente, densidade real e coeficiente de atrito para vários graus de umidade. Quanto ao comportamento mecânico, PERSSON (1987) levantou a hipótese de que a ruptura do material fibroso ocorre quando a tensão de cisalhamento excede o valor crítico. Essa tensão na parede fibrosa pode ser ocasionada pela combinação de tensão de tração na direção da fibra e da tensão de compressão na direção perpendicular à fibra.

OLOSO \& CLARKE (1993), estudando alguns aspectos das propriedades mecânicas da castanha de caju, concluíram que a deformação de ruptura e a energia absorvida no ponto de ruptura aumentavam com o acréscimo do teor de água de castanhas tratadas termicamente no LCC a $190{ }^{\circ} \mathrm{C}$ por $150 \mathrm{~s}$. Embora as propriedades mecânicas do endocarpo e da amêndoa tenham grande relevância no processo de decorticação, essas têm sido pouco estudadas. A determinação dessas propriedades favorece a adequação dos procedimentos atuais de preparação das castanhas, bem como poderá fornecer subsídios para a concepção de mecanismos de máquinas.

Ao se projetarem mecanismos decorticadores, baseados na compressão à determinada deformação específica, alguns fatores devem ser considerados, entre os quais: as alterações nas dimensões das castanhas devido ao tratamento térmico, à fragilidade da casca, à rigidez da amêndoa e à deformação total imposta no momento da decorticação, uma vez que o espaço existente entre a casca e a amêndoa é, via de regra, muito reduzido.

Com base no exposto, este trabalho teve como objetivos comparar as variações das principais dimensões, massa e volume da castanha antes do tratamento térmico e após o tratamento térmico, e avaliar o efeito do tratamento térmico por meio das propriedades mecânicas do endocarpo e das amêndoas, buscando indicar o melhor tratamento térmico.

\section{MATERIAL E MÉTODOS}

\section{Hidratação e tratamento térmico da castanha de caju: preliminares}

Para o estabelecimento dos tempos de hidratação e de residência no LCC para a castanha 'CCP 76', ensaios preliminares foram realizados, tomando como referência o tempo de hidratação da castanha e o tempo da castanha imersa no LCC, praticados pela indústria brasileira de beneficiamento da castanha de caju. Lotes de 25 castanhas de tamanho médio foram submetidos à hidratação, e o tratamento térmico no LCC, em vários intervalos de tempo, com o objetivo de determinar o tratamento mais adequado para a castanha ' $\mathrm{CCP} 76$ '. Em seguida, avaliaram-se os referidos lotes, visualmente, pela cor da amêndoa, observando se o tratamento térmico tinha sido 
excessivo a ponto de comprometer a sua qualidade, procedimento semelhante ao utilizado pela indústria. $\mathrm{O}$ valor encontrado para a hidratação da castanha foi de $86 \mathrm{~h}$ e de $150 \mathrm{~s}$ para o tempo de residência em $\mathrm{LCC}$, a $210^{\circ} \mathrm{C}$. Esses valores foram utilizados como ponto central do planejamento experimental.

\section{Planejamento experimental}

Utilizou-se da superfície de resposta (BARROS NETO et al., 2002) para analisar o efeito combinado do tempo de hidratação e do tempo de residência no LCC. As respostas para identificar o melhor tratamento térmico para a castanha de caju foram os índices de fragilidade do endocarpo, quantificados pela força máxima, deformação específica correspondente e energia de deformação. Os efeitos na amêndoa foram avaliados pela resistência à penetração, por meio da força máxima e da energia de deformação. O delineamento experimental escolhido foi fatorial $2^{2}$, com três pontos na região central e quatro pontos axiais, totalizando 11 pontos experimentais. Os valores experimentais para os níveis das variáveis do tempo de hidratação e do tempo de residência no LCC encontram-se na Tabela 1. A ordem dos fatores foi aleatória.

TABELA 1. Variáveis codificadas e decodificadas do planejamento experimental para a avaliação do efeito combinado de hidratação e do tratamento térmico submetido à castanha de caju ' $\mathrm{CCP} 76$ '. Coded and decoded experimental design variables used to evaluate the combined effect of hydration and thermal treatment on cashew nut 'CCP 76'.

\begin{tabular}{crrcc}
\hline \multirow{2}{*}{ Ensaios } & \multicolumn{2}{c}{ Codificado } & \multicolumn{2}{c}{ Decodificado } \\
\cline { 2 - 5 } & Th $(\mathrm{h})$ & $\operatorname{Tr}(\mathrm{s})$ & $\mathrm{Th}(\mathrm{h})$ & $\operatorname{Tr}(\mathrm{s})$ \\
\hline 1 & -1 & -1 & 93 & 135 \\
2 & 1 & -1 & 79 & 135 \\
3 & -1 & 1 & 93 & 165 \\
4 & 1 & 1 & 86 & 165 \\
5 & 0 & 0 & 86 & 150 \\
6 & 0 & 0 & 86 & 150 \\
7 & 0 & 0 & 72 & 150 \\
8 & -2 & 0 & 100 & 150 \\
9 & 2 & 0 & 86 & 150 \\
10 & 0 & -2 & 86 & 120 \\
11 & 0 & 2 & & 180 \\
\hline
\end{tabular}

Th - tempo de hidratação; Tr - tempo de residência no LLC a $210{ }^{\circ} \mathrm{C}$.

Esse tipo de planejamento estatístico gera um modelo quadrático, em que o valor das variáveis dependentes Y (força máxima, deformação específica e energia) é função das variáveis independentes (tempo de hidratação (Th) e tempo de residência $(\mathrm{Tr})$ no LLC a $210^{\circ} \mathrm{C}$ ), conforme descrito na eq.(1).

$$
\mathrm{Y}=\varphi(\mathrm{Th}, \mathrm{Tr})=\beta_{0}+\beta_{1} \operatorname{Th}+\beta_{2} \operatorname{Tr}+\beta_{11} \operatorname{Th}^{2}+\beta_{22} \operatorname{Tr}^{2}+\beta_{12} \operatorname{Th} \operatorname{Tr}
$$

em que,

Th - tempo de hidratação, horas;

Tr - tempo de residência, segundos;

$\beta_{0}$ - valor populacional da média de todas as respostas do planejamento, e

$\beta_{1}, \beta_{2}, \beta_{11}, \beta_{22}$ e $\beta_{12}$ - valores populacionais dos dois efeitos principais e do efeito de interação, por unidade de Th e de Tr.

Os pontos centrais servem para estimar o erro experimental e determinar a precisão da equação polinomial. Os pontos axiais $( \pm \alpha)$ são utilizados para a ampliação do modelo linear, tornando-o modelo quadrático. Os coeficientes dos modelos de regressão foram obtidos por meio do software STATISTICA ${ }^{\circledR}$ 6.0, sendo considerado preditivo o modelo com regressão significativa 
com 95\% de confiança, falta de ajuste não-significativa com o mesmo nível de confiança e alto valor de $\mathrm{R}^{2}$ (BARROS NETO et al., 2002).

O planejamento estatístico indicará uma região de máxima resposta ou de mínimo, em função do tempo de hidratação e de residência no LCC, ou seja, a região na qual as características mecânicas do material apontem para o que se deseja. Após essa análise, procedeu-se à comparação de médias entre os diversos tratamentos, e o material in natura (testemunha) pelo teste de Tukey $(\mathrm{p} \leq 0,05)$. A escolha do melhor parâmetro (força máxima, deformação específica ou energia) a ser utilizado na caracterização da fragilidade do endocarpo e na rigidez da amêndoa da castanha de caju foi feita em função do menor coeficiente de variação apresentado.

\section{Procedimentos para hidratação e tratamento térmico das castanhas de caju}

Após a identificação do ponto central, procedeu-se à preparação dos lotes para a determinação do teor de água, da caracterização dimensional, das medidas de massa, assim como os ensaios mecânicos para todos os tratamentos. Utilizaram-se castanhas de tamanho médio do clone 'CCP 76', fornecidas pela Embrapa - Agroindústria Tropical, Fortaleza - CE. Os lotes de 25 castanhas in natura foram imersos em água, em recipiente fechado, por período de $2 \mathrm{~h}$. Em seguida, drenou-se a água, e as castanhas foram colocadas em recipiente hermético por um período de repouso durante $72 ; 79 ; 86 ; 93$ e $100 \mathrm{~h}$ para sua hidratação. Após esse tempo, foram submetidas ao tratamento térmico em LCC a $210^{\circ} \mathrm{C}$, por $120 ; 135 ; 150 ; 165$ e $180 \mathrm{~s}$, de acordo com o planejamento experimental. Utilizou-se de 'fritadeira' elétrica comercial (Croydon, modelo F1B-200000-A0), com capacidade de 4,5 L, para o tratamento térmico, sendo o tempo de residência no LCC medido por meio de cronômetro digital (Casio modelo HS10W). Para a determinação do volume, foi considerado o tempo de $86 \mathrm{~h}$ para a hidratação e $150 \mathrm{~s}$ de residência no LCC.

\section{Dimensões, massa e volume das castanhas de caju antes do tratamento térmico e após o tratamento térmico}

Para identificar possíveis variações nas dimensões, na massa e no volume das castanhas, foram realizadas medidas antes e após o tratamento térmico, por meio de paquímetro digital (DIGIMESS), com resolução centesimal, e balança com $0,1 \mathrm{~g}$ de precisão. As dimensões consideradas da castanha, tais como, comprimento, largura e espessura, são representadas, esquematicamente, na Figura 1.
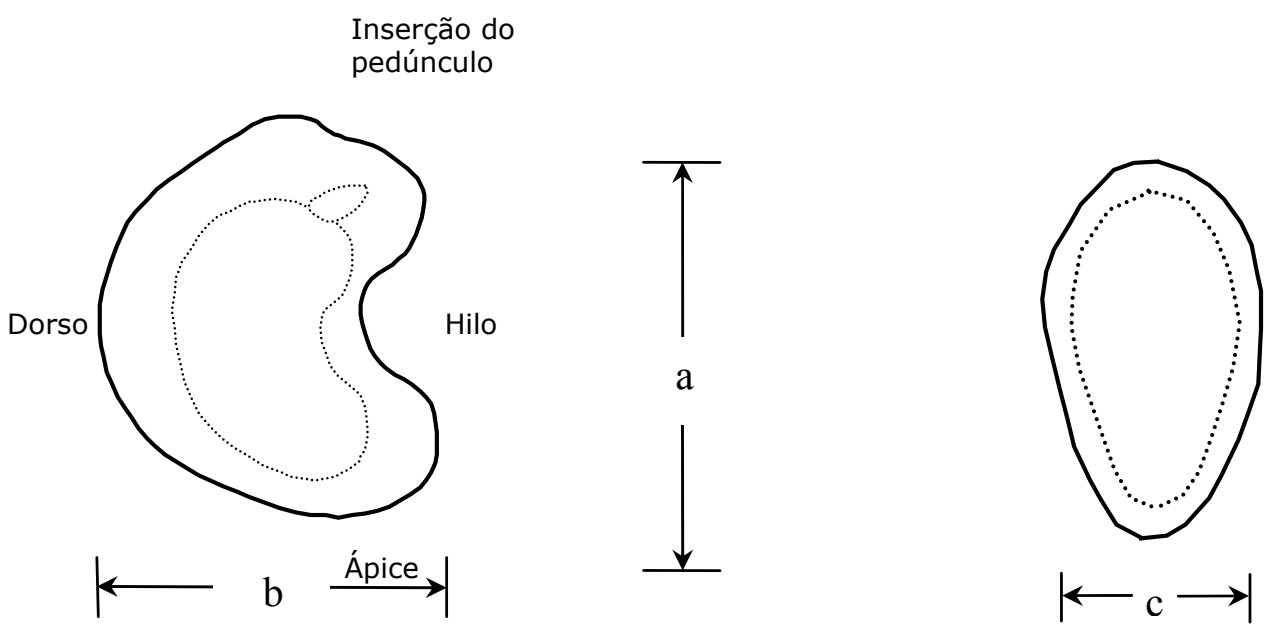

FIGURA 1. Dimensões características da castanha de caju. Comprimento (a), largura (b) e espessura (c). Characteristic dimensions (length (a), width (b) and thickness (c) ) of the cashew nut.

Devido aos cuidados operacionais para a determinação do volume, mensuraram-se 30 castanhas antes e após o tratamento térmico. Cada castanha foi perfurada com um arame de aço, 
pela inserção do pedúnculo, e introduzida, com o auxílio de uma haste de altura regulável, em recipiente contendo quantidade conhecida de água. Mediu-se, então, a massa de cada castanha submersa, por meio das leituras da balança, calculando-se o seu respectivo volume pela relação de densidade (MOHSENIN, 1970). As mesmas castanhas foram submetidas ao tratamento térmico central e submetidas a nova determinação do volume.

\section{Determinação do teor de água}

Para a determinação do teor de água das castanhas, utilizou-se da metodologia proposta por MELO et al. (1998), com estufa com circulação de ar forçado (Fanem 320 SE) e temperatura de $65 \pm 3{ }^{\circ} \mathrm{C}$, até atingir peso constante, com trituração do material, tanto para a amêndoa como para a casca (epicarpo, mesocarpo e endocarpo). Foram realizadas determinações em três repetições com 50 castanhas de cada lote tratado termicamente, utilizando-se de $5 \mathrm{~g}$ de amêndoa triturada e $10 \mathrm{~g}$ de casca triturada para cada repetição. Utilizou-se de balança analítica (Gehaka BG2000), com carga máxima de $2.100 \mathrm{~g}$ e precisão de $0,01 \mathrm{~g}$.

\section{Avaliação do efeito combinado de hidratação e tratamento térmico nas propriedades do endocarpo}

\section{Cisalhamento do endocarpo}

Após os tratamentos térmicos, retirou-se o LCC superficial de cada castanha, com papel toalha, submetendo-as a período de repouso de $2 \mathrm{~h}$ para resfriamento. Cada castanha foi decorticada por meio de dispositivo manual construído para esse fim, apresentado na Figura 2, tomando-se o cuidado de preservar a integridade das amêndoas.
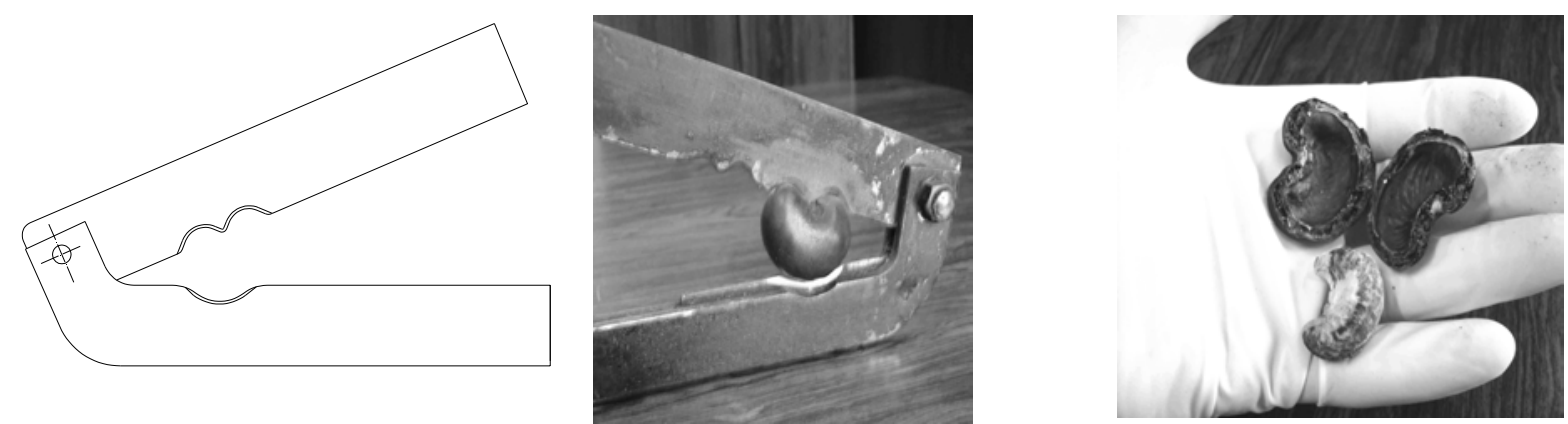

FIGURA 2. Vistas do dispositivo, projetado e construído para a decorticação manual das castanhas de caju, por meio de lâminas afiadas, e castanha aberta sem danos à amêndoa. Device specially designed to cut open the nut shell using sharp blades in order to extract the kernel with no damage.

Após a decorticação, retiraram-se o epicarpo e o mesocarpo por meio de escarificação com faca afiada, isolando-se o endocarpo das demais camadas. Em seguida, posicionou-se o endocarpo no dispositivo para o ensaio de cisalhamento (Figura 3). Para avaliar o efeito dos tratamentos térmicos nas propriedades do endocarpo, concebeu-se um método que avaliasse o nível de fragilidade do endocarpo, por meio de dispositivo de cisalhamento, cujo princípio de funcionamento é mostrado, esquematicamente, nas Figuras 3 e 4 . O dispositivo é composto por ponteira cilíndrica de 3,6 mm de diâmetro (faca), em aço inox, e base com orifício central (contrafaca), em aço 1020 temperado, sendo montado em máquina universal de ensaios (Ottowa Texture Measuring System), equipado com célula de carga de $500 \mathrm{~N}$, sendo utilizados 25 endocarpos para cada tratamento. A avaliação foi feita pelo valor da força máxima, deformação específica correspondente e energia de deformação. Escolheu-se o parâmetro de menor coeficiente de variação para a avaliação da fragilidade do endocarpo. 


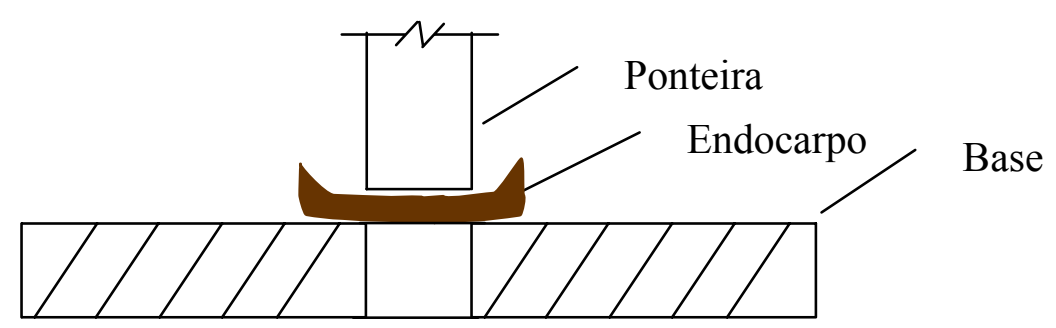

FIGURA 3. Representação esquemática parcial do dispositivo utilizado para o ensaio de cisalhamento de uma parcela do endocarpo de castanhas, evidenciando a ponteira de arestas cortantes e o posicionamento do endocarpo sobre o orifício da base que atua como contrafaca. Partial representation of the endocarp shearing device showing the cylindrical probe with sharp edge, and the orifice acting as counter-shear.

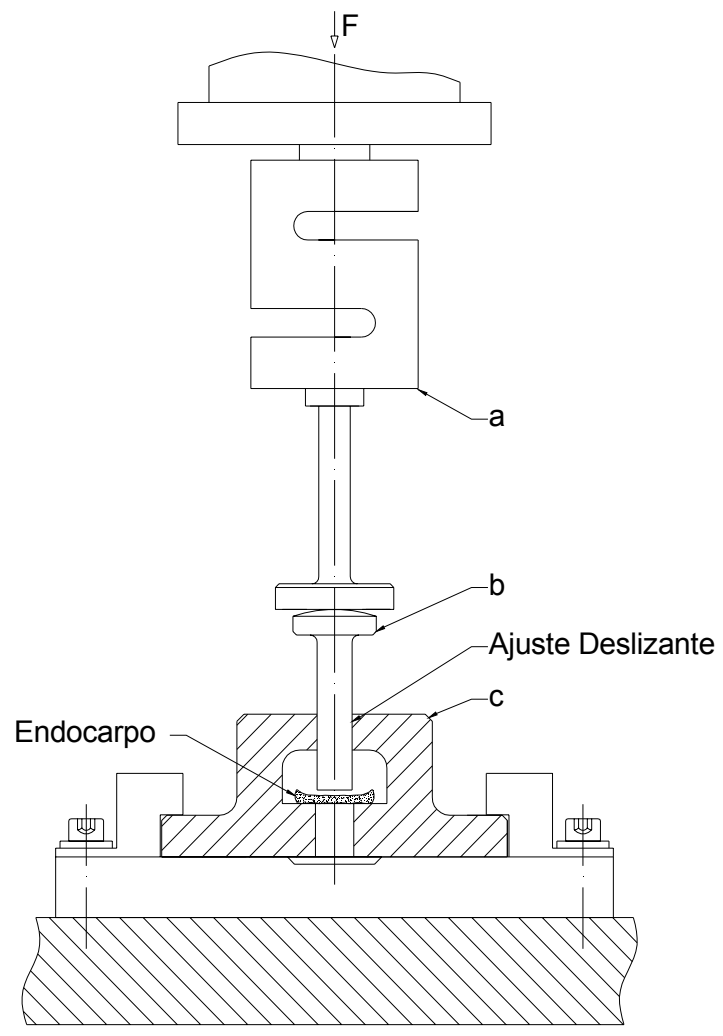

FIGURA 4. Vista do dispositivo de cisalhamento acoplado à máquina universal de ensaios indicando a célula de carga (a), a ponteira (b) e a base plana (c). Shearing device installed in a universal testing machine showing the load cell (a), cylindrical probe (b), and flat base (c).

\section{Avaliação do efeito combinado de hidratação e tratamento térmico nas propriedades da amêndoa}

\section{Penetração da amêndoa}

O ensaio de penetração foi realizado com as amêndoas obtidas, cuidadosamente, por meio de decorticação manual. Utilizou-se de ponteira cilíndrica de $2 \mathrm{~mm}$ de diâmetro (Figura 5) acoplada à máquina universal de ensaios (TEXTURE ANALYSER, Modelo TA 500), com célula de carga de $50 \mathrm{~N}$, à taxa de deformação constante de $0,6 \mathrm{~mm} \mathrm{~s}^{-1}$ e profundidade máxima de $30 \%$ da espessura da amêndoa. Cada espécime foi disposto sobre prato plano e horizontal, perpendicularmente à direção da ponteira e, em seguida, posicionado até o contato com a amêndoa na sua região de maior espessura, iniciando-se o ensaio. Utilizaram-se 25 amêndoas para cada tratamento. A avaliação foi 
feita pelo valor da força máxima e energia de deformação até $30 \%$ de penetração. O parâmetro escolhido para a avaliação da resistência da amêndoa foi o que obteve o menor coeficiente de variação.

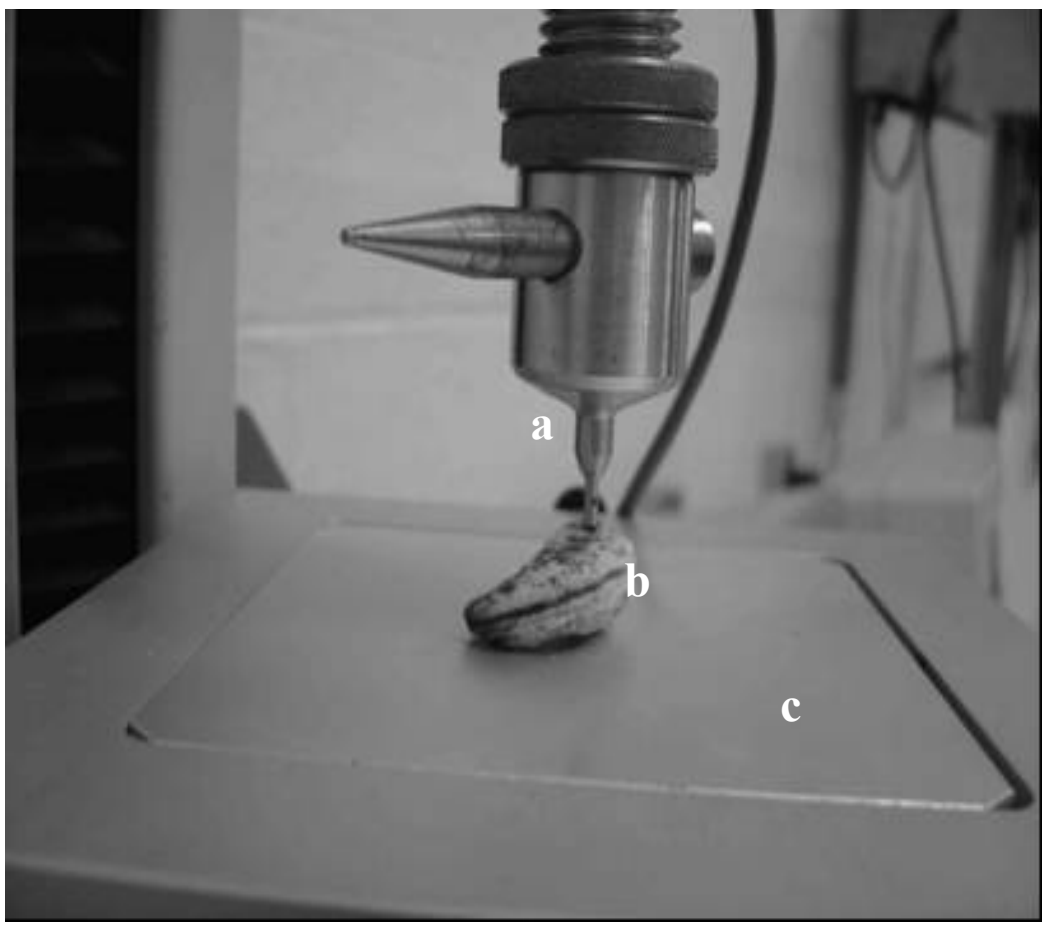

FIGURA 5. Posição da ponteira (a) e amêndoa (b) sobre a base plana (c) durante ensaio de penetração. Relative position of the cylindrical probe (a) and kernel (b) on a flat rigid surface (c).

\section{RESULTADOS E DISCUSSÃO}

\section{Avaliação das dimensões, da massa e do volume antes e após o tratamento térmico}

As dimensões médias das castanhas de caju antes do tratamento térmico e após o tratamento térmico (Tabela 2) apresentaram acréscimo médio de 6,78\% no comprimento, 5,73\% na largura, $5,47 \%$ na espessura, $28,58 \%$ no volume e redução de $24,61 \%$ na massa.

TABELA 2. Médias das dimensões, massa e volume, seguido do seu respectivo desvio-padrão e coeficiente de variação (\%), da castanha de caju antes e após o tratamento térmico com LCC. Cashew nuts average values of dimensions, mass and volume and respective standard deviation and coefficient of variation prior and post heat treatment. The heat treatment was performed by immersing the nut in heated nut shell liquid (NSL).

\begin{tabular}{lcc}
\hline & \multicolumn{2}{c}{ Tratamento Térmico } \\
\cline { 2 - 3 } & Antes & Após \\
\hline Comprimento $\left(10^{-3}\right), \mathrm{m}$ & $33,61 \pm 0,49(1,46)$ & $35,89 \pm 2,32(6,46)$ \\
Largura $\left(10^{-3}\right), \mathrm{m}$ & $27,39 \pm 0,25(0,91)$ & $28,96 \pm 1,99(6,87)$ \\
Espessura $\left(10^{-3}\right), \mathrm{m}$ & $20,65 \pm 0,16(0,77)$ & $21,78 \pm 1,82(8,36)$ \\
Massa $\left(10^{-3}\right), \mathrm{kg}$ & $9,10 \pm 0,21(2,31)$ & $6,86 \pm 1,24(18,08)$ \\
Volume $\left(10^{-6}\right), \mathrm{m}^{3}$ & $8,92 \pm 0,82(9,19)$ & $11,47 \pm 0,93(8,04)$ \\
\hline
\end{tabular}

25 repetições em cada tratamento para comprimento, largura, espessura e massa, e 30 repetições para o volume. Coeficiente de variação entre parênteses (\%)

Observa-se que, após o tratamento térmico, os coeficientes de variação das dimensões e da massa são de 4,5 a 11 vezes maiores, evidenciando que esse tipo de tratamento introduz variabilidade adicional significativa às castanhas. No entanto, para o volume, constatou-se pequena 
redução no coeficiente de variação, de 9,19\% antes do tratamento térmico, para 8,04\% após o tratamento térmico. Em geral, as alterações observadas apontam para a necessidade de classificação após o tratamento térmico quando essa representar parâmetro importante no processo de decorticação da castanha. Quando se aplica deformação limitada na castanha, tendo como objetivo romper o endocarpo e preservar a integridade da amêndoa, essas alterações nas dimensões, na massa e no volume devem ser levadas em consideração para uma boa eficiência no processo de beneficiamento.

\section{Determinação do teor de água}

A determinação do teor de água da casca, composta pelo epicarpo, mesocarpo e endocarpo, e da amêndoa mais a película da castanha de caju, foi realizada triturando-se o material e secando-o em estufa com circulação forçada de ar a $65^{\circ} \mathrm{C}$, até obter peso constante. Esse procedimento mostrou-se eficaz, comparado com os resultados de ensaios preliminares utilizando metodologias tradicionais de determinação da umidade em que houve dificuldade de obter estabilidade nas medidas do peso seco.

Dos valores percentuais médios do teor de água em base seca (b.s.), obtidos (Tabela 3) para a amêndoa e para a casca da castanha de caju para os dez tratamentos (ensaios), observa-se que, para os ensaios com mesmo tempo de hidratação (Th), o teor de água resultante tende a ser maior com a redução do tempo de residência no LCC (Tr). Para os ensaios com o mesmo tempo de residência, o aumento do tempo de hidratação não representa, necessariamente, acréscimo no teor de água. Portanto, o fator que exerce a maior influência no teor de água final é o tempo de residência no LCC a $210^{\circ} \mathrm{C}$.

TABELA 3. Valores percentuais médios do teor de água (b.s.), seguido do seu respectivo desviopadrão e coeficiente de variação (\%) para amêndoa e casca (epicarpo, mesocarpo e endocarpo) da castanha de caju 'CCP 76' submetida às várias combinações de tempo de hidratação (Th) e tempo de residência (Tr) no LCC, a $200{ }^{\circ} \mathrm{C}$. Moisture content (db) average values followed by standard deviation and coefficient of variation (\%) for kernel and shell (epicarp, mesocarp and endocarp) of cashew nut ' $C C P$ 76' subjected to various combinations of hydration times (Th) and dwell time (Tr) in nut shell liquid at $200^{\circ} \mathrm{C}$.

\begin{tabular}{|c|c|c|c|c|c|c|}
\hline Ensaios & $\begin{array}{l}\text { Th } \\
\text { (h) }\end{array}$ & $\begin{array}{l}\mathrm{Tr} \\
(\mathrm{s})\end{array}$ & Ensaios & $\begin{array}{l}\text { Teor de Água da } \\
\text { Amêndoa (\%) }\end{array}$ & Ensaios & $\begin{array}{c}\text { Teor de Água da Casca } \\
(\%)\end{array}$ \\
\hline 1 & 79 & 135 & 9 & $6,72 \pm 0,12(1,79) a^{* * *}$ & 12 & $7,12 \pm 0,13(1,83) \mathrm{a}$ \\
\hline 2 & 93 & 135 & 10 & $6,65 \pm 0,12(1,80)$ ba & 2 & $4,60 \pm 0,50(10,87) b$ \\
\hline 3 & 79 & 165 & 8 & $6,53 \pm 0,11(1,68)$ bac & 10 & $4,54 \pm 0,43(9,47) b$ \\
\hline 4 & 93 & 165 & 1 & $5,93 \pm 0,08(1,35) b d c$ & 8 & $4,04 \pm 0,44(10,89) \mathrm{cb}$ \\
\hline $5^{*}$ & 86 & 150 & 12 & $5,84 \pm 0,09(1,54) \mathrm{dc}$ & 1 & $4,03 \pm 0,40(9,93) \mathrm{cb}$ \\
\hline 8 & 72 & 150 & 2 & $5,81 \pm 0,08(1,38) \mathrm{dc}$ & 5 & $3,38 \pm 0,10(2,96) \mathrm{cd}$ \\
\hline 9 & 100 & 150 & 3 & $5,75 \pm 0,19(3,30) d$ & 3 & $3,35 \pm 0,43(12,84) \mathrm{cd}$ \\
\hline 10 & 86 & 120 & 5 & $5,54 \pm 0,24(4,33) d$ & 9 & $3,20 \pm 0,17(5,31) \mathrm{cd}$ \\
\hline 11 & 86 & 180 & 4 & $5,42 \pm 0,02(0,37) d$ & 11 & $2,50 \pm 0,04(1,60) d$ \\
\hline $12 * *$ & 0 & 0 & 11 & $5,25 \pm 0,74(14,10) d$ & 4 & $2,49 \pm 0,14(5,62) d$ \\
\hline
\end{tabular}

* Média de três repetições do ponto central do planejamento experimental. O número de repetições nos demais ensaios é três. $* *$ castanha in natura. $* * *$ média acompanhada de mesma letra, na coluna, não apresenta diferença significativa (Tukey, $\mathrm{p}<0,05$ )

Para os ensaios 1; 8; 9 e 10, ocorreu hidratação da amêndoa; para os demais, ocorreu perda de água, comparados ao teor médio da amêndoa in natura (ensaio 12). Observou-se maior ganho de água para as amêndoas do ensaio 9, com 13,1\% de aumento no teor médio, e a maior perda foi de $10 \%$, correspondente ao ensaio 11 . Os tratamentos $9 ; 10$ e 8 diferenciaram-se significativamente 
dos tratamentos $3 ; 5 ; 4$ e 11, evidenciando que os ensaios afetaram distintamente a absorção e a dessorção de água da amêndoa.

BORGES \& PELEG (1997) afirmaram que a presença de óleo nas nozes parece ser a principal causa da ausência de fragilidade, sendo o fator limitante da absorção ou dessorção de água, tornando-as mais ou menos rígidas. A afirmação é válida para a amêndoa da castanha de caju que, para os tratamentos considerados, tanto na absorção como na dessorção, apresentaram alterações relativamente pequenas no teor de água, comparadas àquelas sofridas pela casca. A perda de água pela amêndoa, promovida por alguns dos tratamentos, pode ser inconveniente na decorticação, pois pode enrijecê-la (ARAÚJO, 2005).

Observou-se perda de água na casca para todos os ensaios. A casca in natura (ensaio 12) apresentou teor de água médio que variou de 35,4\% a 65,1\% superior, e diferiu significativamente (Tukey, $\mathrm{p} \leq 0,05$ ) dos demais tratamentos. Esse resultado aponta para possíveis alterações no comportamento mecânico da casca, pois a perda d'água pode torná-la mais frágil.

A comparação de médias revelou ser complexa a interação entre o tempo de hidratação e o tempo de residência em LCC e será analisada a seguir por meio da metodologia de superfície de resposta.

\section{Efeito combinado de hidratação e tratamento térmico nas propriedades do endocarpo}

Para a avaliação do efeito combinado de hidratação e tratamento térmico nas propriedades mecânicas do endocarpo, elegeu-se como índice de resistência do endocarpo a deformação específica na força máxima, por apresentar o menor coeficiente de variação. Duas curvas características das relações força-deformação, obtidas no ensaio de cisalhamento do endocarpo, ensaios 1 e 12, mostradas na Figura 6, exemplificam os distintos comportamentos observados. O endocarpo submetido à hidratação e ao tratamento térmico (ensaio 1) apresentou menores valores de força máxima e de deformação específica na força máxima, indicando que o endocarpo se tornou mais frágil (CHIAVERINI, 1986). Para os demais tratamentos, verificou-se comportamento semelhante.

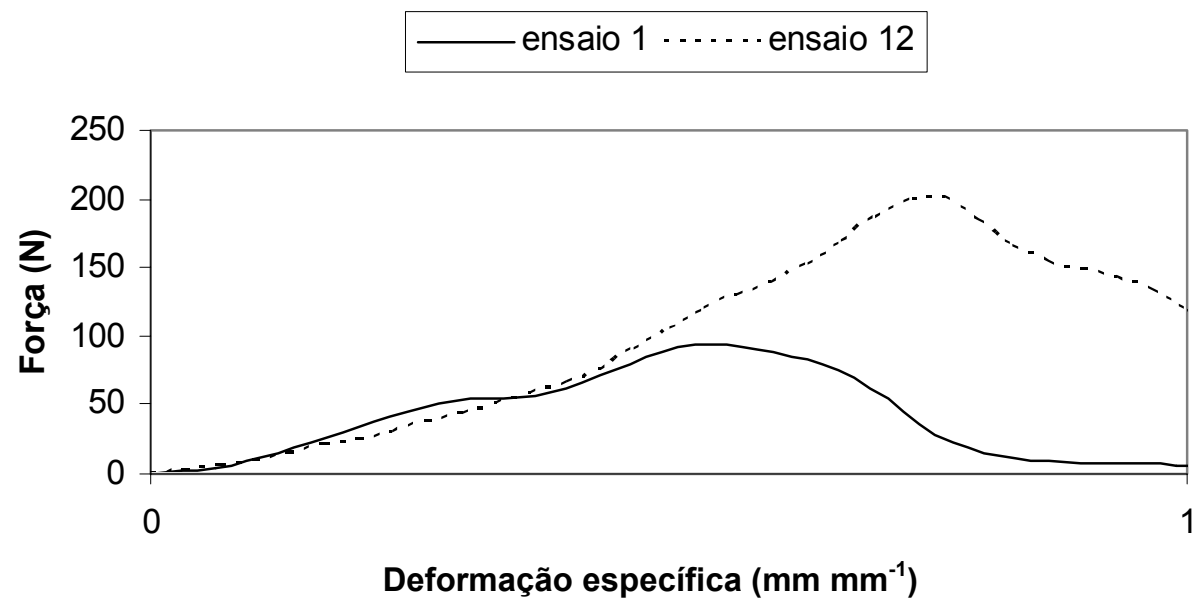

FIGURA 6. Relações força-deformação características, para o ensaio de cisalhamento do endocarpo com tratamento térmico (ensaio 1) e sem tratamento térmico (ensaio 12). Forcedeformation characteristic curves obtained from the endocarp shearing tests using a heat treated specimen (1) and a non-treated one (12).

Os valores médios obtidos para a deformação específica (Tabela 4) apresentaram menores coeficientes de variação que a força máxima e a energia de deformação e, por isso, foram utilizados como índice de resistência do endocarpo. 
TABELA 4. Valores médios de deformação específica $\left(\mathrm{mm} \mathrm{mm}^{-1}\right)$ na força máxima obtidos nos ensaios de cisalhamento do endocarpo em diversos tratamentos. Specific deformation $\left(\mathrm{mm} \mathrm{mm}^{-1}\right)$ average values at maximum force obtained from shearing tests for various endocarp treatments.

\begin{tabular}{cccc}
\hline Ensaio & Deformação Específica $\left(\mathrm{mm} \mathrm{mm}^{-1}\right)$ & Ensaio & Deformação Específica $\left(\mathrm{mm} \mathrm{mm}^{-1}\right)$ \\
\hline 1 & $0,71(23)^{*}$ & 8 & $0,78(9)$ \\
2 & $0,77(17)$ & 9 & $0,79(15)$ \\
3 & $0,76(19)$ & 10 & $0,75(23)$ \\
4 & $0,78(14)$ & 11 & $0,79(16)$ \\
$5^{* *}$ & $0,84(8)$ & 12 & $0,75(18)$ \\
\hline
\end{tabular}

*coeficiente de variação (\%); ** ponto central do planejamento experimental; *** castanha in natura.

Observam-se, na Tabela 5, os efeitos estimados, erro puro e grau de significância da deformação específica para o cisalhamento do endocarpo. Os efeitos estimados nos diferentes fatores estudados não apresentaram diferenças estatísticas $(\mathrm{p} \leq 0,05)$. Para o tempo de hidratação e o tempo de residência, no modelo linear, o efeito teve sinal positivo, indicando que a resposta da deformação específica deve ser maior quando se aumenta o tempo de hidratação ou de residência no LCC. Para a interação, o efeito teve sinal negativo, apontando para uma resposta menor da deformação específica quando se aumenta a interação dos dois fatores estudados. O tempo de umidificação no modelo quadrático foi o que apresentou maior valor de efeito na resposta da deformação específica. Quanto maior o tempo de hidratação, menores as respostas de deformação específica.

TABELA 5. Efeitos estimados, erro puro e grau de significância estatística para a deformação específica no ensaio de cisalhamento do endocarpo. Estimated effects, pure error and level of statistical significance for the endocarp specific deformation under shearing tests.

\begin{tabular}{lrcc}
\hline \multicolumn{1}{c}{ Fonte de Variação } & Efeito & Erro Puro & $\mathrm{p}$ \\
\hline Média global & 0,8174 & 0,0347 & $0,0018^{*}$ \\
Tempo de umidificação (L) & 0,0180 & 0,0390 & 0,6893 \\
Tempo de umidificação (Q) & $-0,0264$ & 0,0308 & 0,4810 \\
Tempo de residência (L) & 0,0257 & 0,0390 & 0,5782 \\
Tempo de residência (Q) & $-0,0320$ & 0,0308 & 0,4071 \\
Interação (L) & $-0,0222$ & 0,0676 & 0,7736 \\
\hline
\end{tabular}

* significativo a $5 \%$ de probabilidade; L - modelo linear; Q - modelo quadrático.

Em virtude da dificuldade de extrair conclusões práticas com essa análise, buscou-se identificar, pelo método de superfície de resposta (BARROS NETO et al., 2002), possível tendência dos ensaios em tornar o endocarpo mais frágil, isto é, com menores valores de deformação específica na força máxima. Na superfície de resposta (Figura 7a), a região do ótimo, no sentido das menores deformações, é representada pela cor verde. A curva de contorno da superfície de resposta (Figura 7b) permite identificar os ensaios 1; 8 e 10, assim como os ensaios 11; 4 e 9, como os mais próximos da região de ótimo. Evidencia-se que o ensaio $11(\mathrm{Th}=86 \mathrm{~h}$ e $\mathrm{Tr}=180 \mathrm{~h})$, com o maior tempo de residência no LCC, não proporcionou, significativamente, um endocarpo mais frágil, ou mesmo se aproximou mais da região de mínimos. As demais combinações de Th e Tr também não apresentam tendência, revelando que o efeito dessas combinações são interações e alterações complexas nos tecidos do endocarpo. 

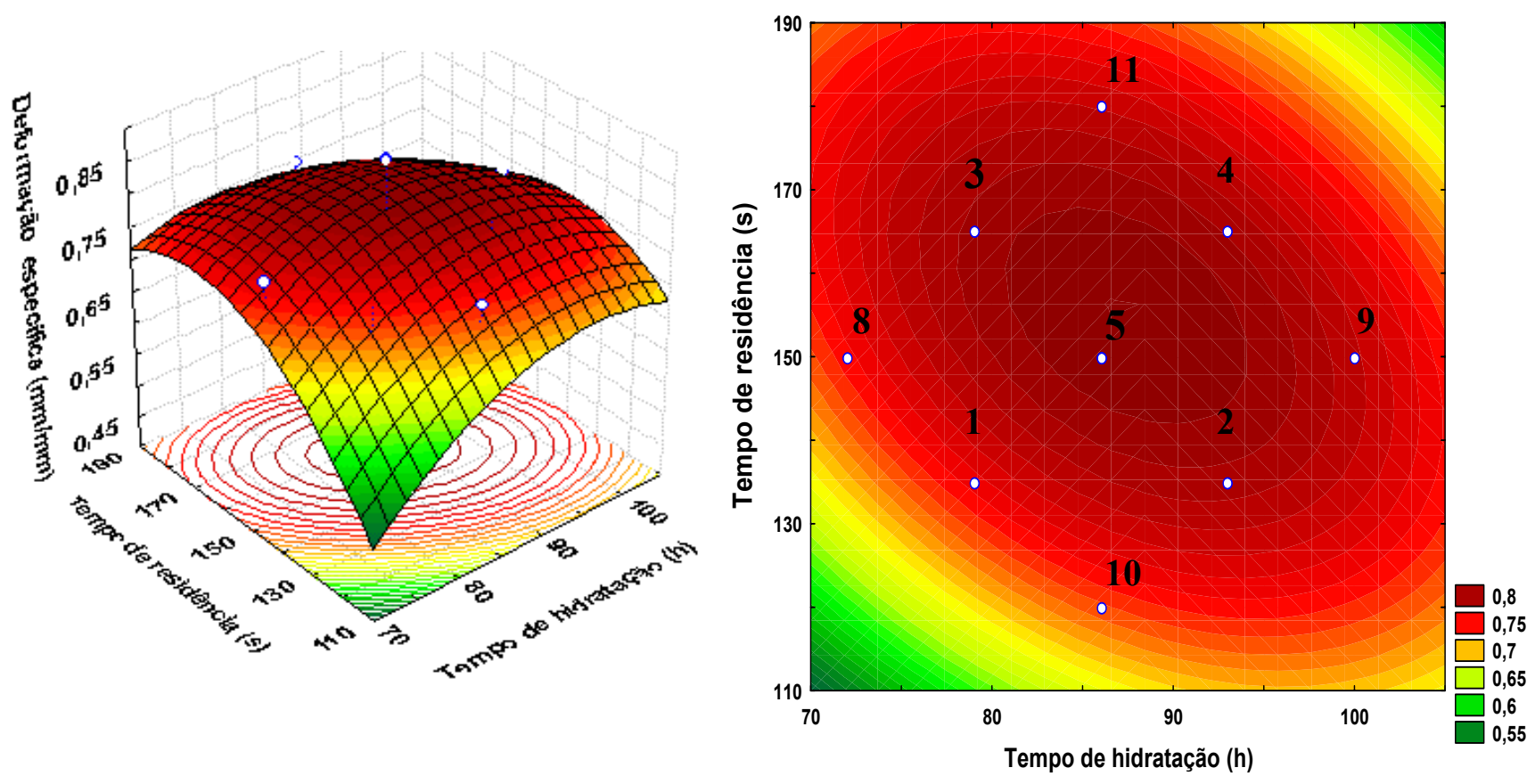

FIGURA 7. Tendência da superfície de resposta e curva de contorno para a deformação específica no endocarpo, em função do tempo de hidratação e do tempo de residência. Surface response tendency and contour curve for specific deformation of the endocarp as a function of hydration time and dwell time.

\section{Efeito combinado de hidratação e do tratamento térmico nas propriedades da amêndoa}

Para a avaliação do efeito combinado de hidratação e do tratamento térmico nas propriedades da amêndoa, utilizou-se, como índice de resistência da amêndoa, da energia de deformação a 30\% de deformação específica por apresentar menor coeficiente de variação. Na Figura 8, mostram-se duas curvas características das relações força-deformação obtidas no ensaio de penetração da amêndoa, sendo uma delas com tratamento térmico (ensaio 1) e a outra sem tratamento térmico (ensaio 12). A maioria das curvas, como ilustra a Figura 8, apresentou comportamento monotônico crescente com maior rigidez no início da penetração, identificado pela maior inclinação da curva. Para os demais tratamentos, o comportamento foi semelhante.

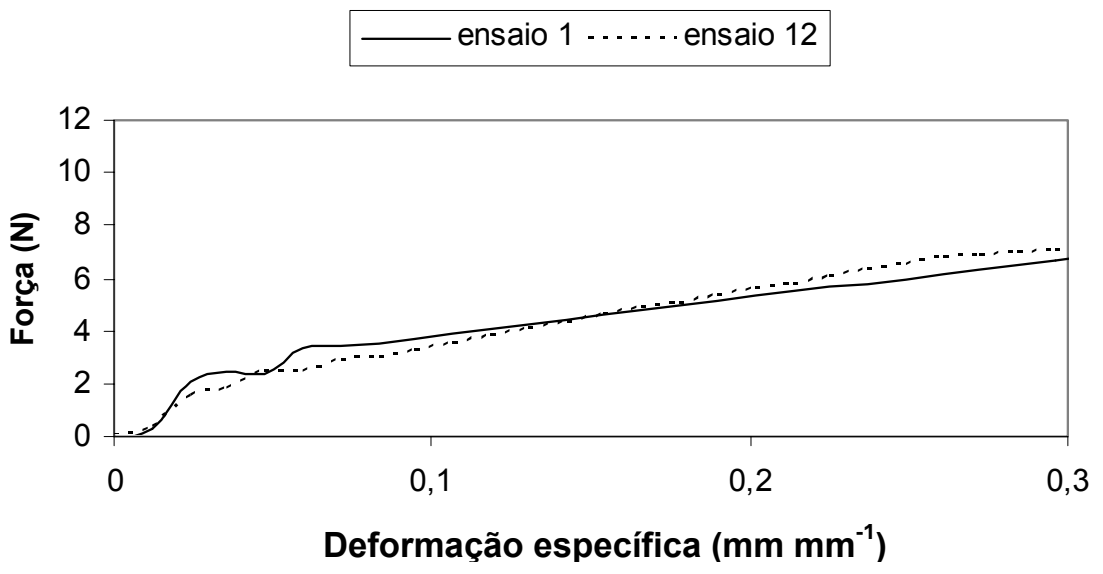

FIGURA 8. Relações força-deformação característica, para o ensaio de penetração da amêndoa com tratamento térmico (ensaio 1) e sem tratamento térmico (ensaio 12). Forcedeformation characteristic curves obtained from the kernel penetration tests using a heat treated specimen (1) and a non-treated one (12). 
A amêndoa mais úmida deveria apresentar menor resistência, implicando menores níveis de força durante a penetração, tendência essa identificada por inspeção visual das curvas.

Os valores médios da energia de deformação até $30 \%$ da deformação específica, obtidos nos ensaios de penetração e respectivos coeficientes de variação, são mostrados na Tabela 6, com seus respectivos coeficientes de variação para os ensaios realizados. Os coeficientes de variação para a energia de deformação foram significativamente menores que aqueles obtidos para a força máxima e, por essa razão, foram escolhidos para a comparação.

TABELA 6. Valores médios de energia de deformação $(\mathrm{J})$ da amêndoa da castanha de caju submetida ao ensaio de penetração. Average values of the cashew kernel deformation energy $(J)$ subjected to penetration tests.

\begin{tabular}{cccc}
\hline Ensaio & Energia $(\mathrm{J}) \times 10^{-2}$ & Ensaio & Energia $(\mathrm{J}) \times 10^{-2}$ \\
\hline 1 & $2,26(23)^{*}$ & 8 & $3,13(19)$ \\
2 & $2,60(15)$ & 9 & $2,45(18)$ \\
3 & $2,95(21)$ & 10 & $2,51(14)$ \\
4 & $2,57(14)$ & 11 & $2,65(24)$ \\
$5^{*}$ & $2,66(4)$ & $12^{* * *}$ & $2,27(22)$ \\
\hline
\end{tabular}

*coeficiente de variação $(\%) ; * *$ valor médio do ponto central do planejamento experimental; *** castanha in natura.

No entanto, não foram observadas diferenças pela análise de variância $(\mathrm{p} \leq 0,05)$ nos fatores estudados, como é observado nos resultados da Tabela 7, que apresenta o efeito estimado, erro puro e grau de significância para a energia do ensaio de penetração da amêndoa. Os efeitos estimados para todos os fatores estudados foram não-significativos a $95 \%$ de confiança $(p \leq 0,05)$. O tempo de hidratação e a interação no modelo linear tiveram efeitos negativos, indicando que a energia deve ser menor quando se aumenta o tempo de umidificação ou a sua interação. $O$ tempo de residência no modelo linear teve efeito positivo, apontando para energia menor quando o tempo de residência no LCC é maior. Maiores tempos de residência proporcionam maior rigidez da amêndoa e, conseqüentemente, maiores riscos de danos.

TABELA 7. Efeitos estimados, erro puro e grau de significância estatística para a energia do ensaio de penetração da amêndoa. Estimated effects, pure error and statistical level of significance for energy values after the penetration test of the kernel.

\begin{tabular}{lccc}
\hline \multicolumn{1}{c}{ Fonte de Variação } & Efeito & Erro-Padrão & $\mathrm{p}$ \\
\hline Média global & 0,0262 & 0,0006 & $0,0005^{*}$ \\
Tempo de umidificação (L) & $-0,0023$ & 0,0007 & $0,0723 \mathrm{~ns}$ \\
Tempo de umidificação (Q) & 0,0007 & 0,0005 & $0,3097 \mathrm{~ns}$ \\
Tempo de residência (L) & 0,0016 & 0,0007 & $0,1423 \mathrm{~ns}$ \\
Tempo de residência (Q) & $-0,0003$ & 0,0005 & $0,5793 \mathrm{~ns}$ \\
Interação (L) & $-0,0036$ & 0,0011 & $0,0887 \mathrm{~ns}$ \\
\hline
\end{tabular}

* significativo a 5\% de probabilidade; ns - não-significativo; L - modelo linear; Q - modelo quadrático

Devido a isso, e semelhantemente ao estudo do comportamento do endocarpo, apresentam-se, na Figura 9, a tendência da superfície de resposta e a curva de contorno para a energia na amêndoa em função do tempo de hidratação e do tempo de residência no LCC. Os níveis de menor energia estão indicados pelas regiões em verde, crescentes no sentido dos tons mais escuros, e evidenciam tendências opostas, isto é, ensaios 1 e 10, com menores tempos de hidratação e de residência, apresentam tendência semelhante aos ensaios 4; 9 e 11, que possuem os maiores períodos de hidratação e de residência. Os ensaios 8 e 3 foram os que mais se distanciaram das regiões de menor energia, indicando maior resistência da amêndoa à penetração. Os ensaios 5 e 2, com tempos intermediários de hidratação e residência, também exibiram posição intermediária na região de menores índices de energia. Esses resultados evidenciam não haver tendência única das diversas 
combinações de tempos de hidratação e de residência na resistência à penetração da amêndoa. Evidenciam, também, que maiores valores do índice de resistência não estão somente associados ao conteúdo de água da amêndoa, na faixa de variação considerada.
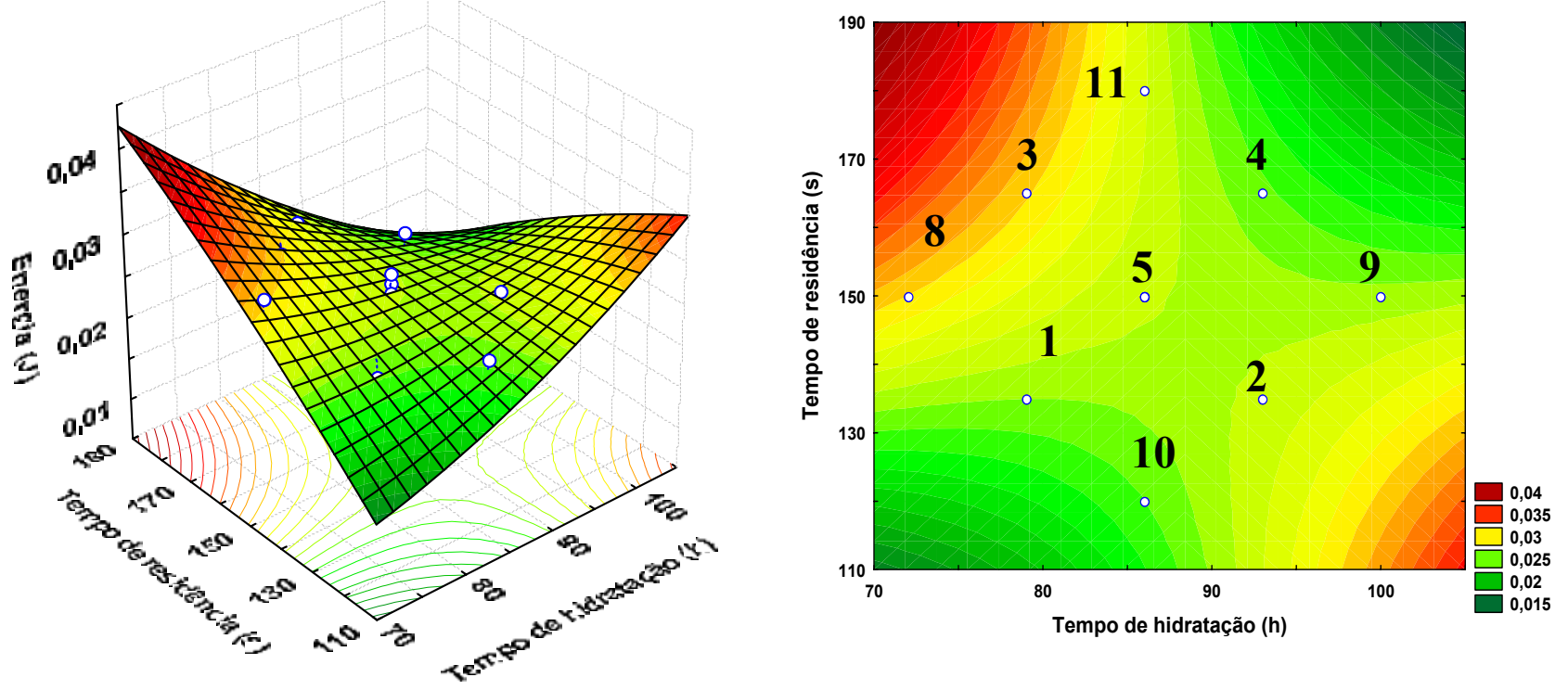

FIGURA 9. Tendência da superfície de resposta e curva de contorno para a energia na amêndoa, em função do tempo de hidratação e do tempo de residência no LCC. Surface response curve tendency and contour curve for the kernel specific deformation as a function of hydration time and dwell time in NSL.

\section{Efeito combinado de hidratação e tratamento térmico para a decorticação da castanha}

Combinando as duas análises anteriores, os tratamentos cujos tempos de hidratação e de residência no LCC que tendem a produzir, simultaneamente, endocarpo mais frágil e amêndoa menos rígida, condições desejadas na decorticação da castanha de caju, são os tratamentos 1; 10; 4 e 9.

\section{CONCLUSÕES}

As diferenças dimensionais encontradas após a preparação das castanhas de caju ' $\mathrm{CCP}$ 76' indicam a necessidade de classificação após o tratamento térmico. Os períodos de 79; 86; 93 e 100 h de hidratação combinados, respectivamente, com os períodos de $135 ; 120 ; 165$ e 150 s de residência no LCC a $210^{\circ} \mathrm{C}$ representam as combinações que tendem a produzir, simultaneamente, endocarpo mais frágil e amêndoa menos rígida, que são características desejáveis para a decorticação.

\section{AGRADECIMENTOS}

À Embrapa - Agroindústria Tropical, Fortaleza - CE, pelo apoio e envio do material; ao Laboratório de Propriedades Mecânicas dos Materiais Biológicos e à Oficina Mecânica da Faculdade de Engenharia Agrícola, UNICAMP, Campinas - SP, pelo apoio técnico durante a realização dos ensaios e construção dos dispositivos, e ao aluno Acássio Farias Barbosa, pelo auxílio nos desenhos.

\section{REFERÊNCIAS}

ARAUJO, M. C. Caracterização mecânica da castanha de caju (Anacardium ocidentale L.) para fins de beneficiamento e desenvolvimento de decorticador de cilindros rotativos. $108 \mathrm{f}$. Dissertação (Mestrado em Engenharia Agrícola) - Universidade Estadual de Campinas, Faculdade de Engenharia Agrícola, Campinas, 2005. 
BALASUBRAMANIAN; D. Physical properties of raw cashew nut. Journal of Agricultural Research, v.78, n.3, p.291-7, 2001.

BARROS NETO, B.; SCARMINIO, I.S.; BRUNS, R.E. Como fazer experimentos - pesquisa e desenvolvimento na ciência e na indústria. Campinas: Editora da Unicamp, 2002. 401 p.

BORGES, A.; PELEG M. Effect of water activity on the mechanical properties of selected legumes and nuts. Journal Science Food Agricultural, New York, v.75, p.463-71, 1997.

CHIAVERINI, V. Tecnologia mecânica. 2. ed. São Paulo: Makron Books, 1986. 266 p.

MELO, M.L.P.; MAIA, G.A.; SILVA, A.P.V.; OLIVEIRA, G.S.F.; FIGUEIREDO, R.W. Caracterização físico-química da amêndoa da castanha de caju (Anacardium occidentale L.) crua e tostada. Ciência e Tecnologia de Alimentos, Campinas, v.18, n.2, p.184-7, 1998.

MOHSENIN, N. N. Physical properties of plant and animal materials: structure, physycal characteristics and mechanical properties. New York: Gordon and Breach Science Publishers, 1970. $891 \mathrm{p}$.

OLOSO, A.O.; CLARKE, B. Some aspects of strength properties of cashew nuts. Journal of Agricultural Engineering Research, Silsoe, v.55, n.1, p.27-43, 1993.

PERSSON, S. Mechanics of cutting plant material. St. Joseph: ASAE Technical Editor, 1987. $288 \mathrm{p}$. 